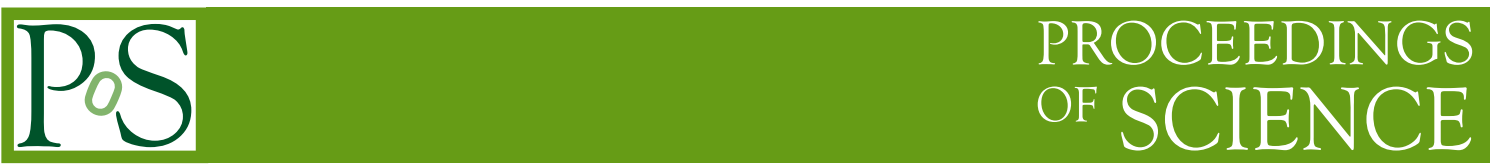

\title{
QCD and low-x physics at a Large Hadron electron Collider
}

\author{
Paul Laycock* \\ $\dagger$ \\ University of Liverpool \\ E-mail: laycock@hep.ph.liv.ac.uk
}

The Large Hadron electron Collider $(\mathrm{LHeC})$ is a proposed facility which will exploit the new world of energy and intensity offered by the LHC for electron-proton scattering, through the addition of a new electron accelerator. This contribution, which is derived from the draft CERNECFA-NuPECC Conceptual Design report (due for release in 2011), addresses the expected impact of the $\mathrm{LHeC}$ precision and extended kinematic range for low Bjorken-x and diffractive physics, and detailed simulation studies and prospects for high precision QCD and electroweak fits. Numerous observables which are sensitive to the expected low-x saturation of the parton densities are explored. These include the inclusive electron-proton scattering cross section and the related structure functions $F_{2}$ and $F_{L}$, as well as exclusive processes such as deeply-virtual Compton scattering and quasi-elastic heavy vector meson production and diffractive virtual photon dissociation. With a hundred times the luminosity that was achieved at HERA, salient expectations for the $\mathrm{LHeC}$ include the complete determination of all light and heavy quark parton distributions for the first time, the high precision extraction of the gluon density, the determination of the strong coupling constant to per-mil accuracy and the precision study of the running of the electroweak mixing angle.

The 2011 Europhysics Conference on High Energy Physics-HEP 2011,

July 21-27, 2011

Grenoble, Rhône-Alpes France

\footnotetext{
* Speaker.

$\dagger$ on behalf of the LHeC Study Group.
} 

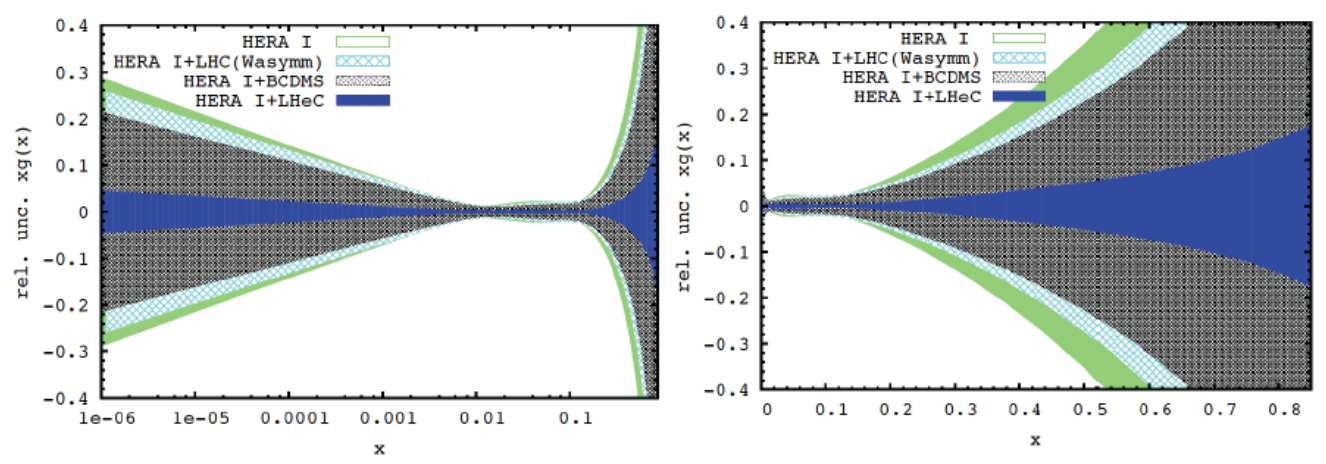

Figure 1: The relative uncertainty on the gluon on a linear and $\log x$ scale, in scenarios with and without the LHeC.

\section{Introduction}

The Large Hadron electron Collider ( $\mathrm{LHeC}$ ) concept is to collide a new high energy electron beam with an existing LHC proton or ion beam, while simultaneously running the full LHC physics programme. The Conceptual Design Report is currently under review from CERN-appointed referees. Only a selection of highlights are presented here, the reader is strongly encouraged to find further information on the $\mathrm{LHeC}$ web site [1].

\section{Physics Highlights}

Among the highlights of the $\mathrm{LHeC}$ is its impact on our understanding of proton structure ${ }^{1}$. The expected size of the final dataset is of order $100 \mathrm{fb}^{-1}$, some two orders of magnitude more than HERA. A challenging but feasible large detector acceptance down to $1^{\circ}$ gives a kinematic extension of a factor of $\approx 20$ in $x$ and $Q^{2}$ with respect to the HERA experiments. The implications for proton structure measurements are correspondingly large. For example, the uncertainty on the gluon PDF, shown in Figure 1, would be greatly improved, reaching percent level precision across the full kinematic range.

Similar levels of improvement are expected in the valence quark distributions, and also on the strange quark content of the proton thanks to high precision charged current measurements. The improvement in kinematic coverage and precision of heavy flavour measurements, courtesy of silicon tracking based on existing technologies, would allow much progress in our understanding of heavy flavour quarks in the proton. The precision of the data will be sensitive to different theoretical approaches to the treatment of heavy flavours in fits and allow the massive and massless approaches to be distinguished. Such fits would be able to constrain the strong coupling constant to the per-mil level of precision.

\footnotetext{
${ }^{1}$ It should be noted that, thanks to the electron-ion colliding mode of operation, the improvement in our knowledge of nucleon structure coming from the $\mathrm{LHeC}$ can be compared to the impact of HERA on proton structure.
} 
Pseudo-data from AAMS09 (BK + running coupling)

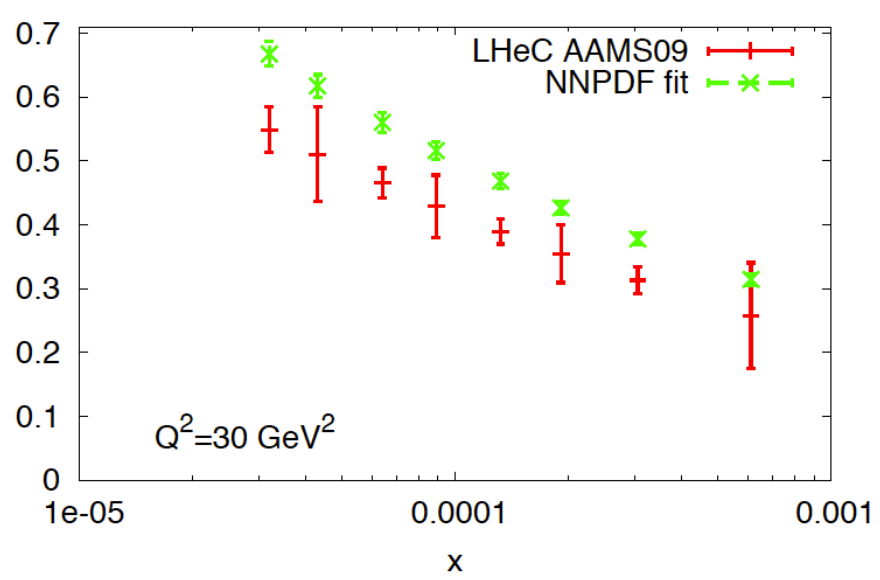

Figure 2: The longitudinal structure function $F_{L}$ from pseudo-data which includes saturation (AAMS09) compared to a fit to the same data which does not allow for saturation.

\subsection{Parton Saturation}

The rise of the gluon PDF at low $x$ cannot continue forever without violating unitarity, but conclusive proof of saturation has yet to be seen. Thanks to its kinematic coverage, the LHeC should be able to resolve this. Figure 2 shows longitudinal structure function pseudo-data generated using a model which includes saturation (AAMS09), compared to a fit to the same data which does not allow for saturation (NNPDF Fit). The fit clearly fails to describe the data.

\subsection{Diffraction}

A related phenomenon is diffraction, where colour-singlet configurations dominate. Exploiting the rapidity gap toplogy, the experimental precision will again be improved, while the larger centre of mass energy means that larger-mass diffractive final states can be accessed than at HERA. This opens up the possibility to measure diffractively produced $W$ and $Z$ bosons, where the $W$ in particular will test the quark flavour symmetry assumptions normally made when modelling diffraction. Exclusive diffractive vector meson production, especially the $J / \Psi$, has long been used as a test of perturbative QCD and to look for saturation effects. The large extension in the kinematic range may mean that exclusive $J / \Psi$ data will be able to distinguish between models of saturation.

\section{Conclusion}

The $\mathrm{LHeC}$ would provide a new window on QCD and low $x$ physics, providing data with a kinematic range and precision that will greatly improve our knowledge of both.

\section{References}

[1] $\mathrm{LHeC}$ web site cern.ch/lhec 